\title{
Psychometric Evaluation of the Beck Anxiety Inventory: A Sample With Sleep-Disordered Breathing
}

\author{
Stacy D. Sanford \\ St. Jude Children's Research Hospital \\ Andrew J. Bush \\ University of Tennessee Health Science Center \\ Kristen C. Stone \\ Warren Alpert Medical School of Brown University \\ Kenneth L. Lichstein \\ The University of Alabama \\ Neal Aguillard \\ Methodist Healthcare
}

\begin{abstract}
This study aimed to document the psychometric properties of the Beck Anxiety Inventory (BAI) within a population with sleep-disordered breathing (SDB), given concerns about overlapping symptomatology between anxiety and sleep apnea. Results supported good internal consistency and convergent and discriminant validity for the BAI and a single-factor solution for men, women, and the total sample. Women had higher scores than men, and discriminant analyses differentiated men from women based on item responses. The BAI has acceptable reliability and validity within a SDB population, supporting its use as a gauge of anxiety severity in individuals with SDB. This is a preliminary study to measure anxiety severity in SDB; future research is needed to determine the utility of the BAI as a diagnostic screener.
\end{abstract}

Correspondence should be addressed to Stacy D. Sanford, Robert H. Lurie Comprehensive Cancer Center, 675 N. St. Clair St. Suite 21-100, Chicago, IL 60611. E-mail: ssanford@nmff.org 
Sleep-disordered breathing (SDB) is characterized by a spectrum of respiratory abnormalities that occur during sleep, with obstructive sleep apnea being the most common. SDB is associated with impairments in quality of life and emotional functioning including symptoms of anxiety (for literature reviews, see Andrews \& Oei, 2004; Haynes, 2005). One study found anxiety disorders to be more prevalent among veterans with sleep apnea than those without, even after controlling for other chronic medical conditions (Sharafkhaneh, Giray, Richardson, Young, \& Hirshkowitz, 2005). Anxiety levels have been found to positively correlate with the apnea-hypopnea index, whereas treatment of sleep apnea has been associated with decreases in anxiety (Borak, Cieslicki, Koziej, Matuszewski, \& Zielinski, 1996; Sanchez, Buela-Casal, Bermudez, \& CasasMaldonado, 2001). This suggests a relation between apnea severity and anxiety symptoms. With this in mind, a routine assessment of anxiety symptoms is imperative for sound multidisciplinary treatment of SDB, but measuring anxiety in populations with SDB might be complicated by overlap between the symptoms of anxiety and sleep disorders. For example, the Beck Anxiety Inventory (BAI; Beck, Epstein, Brown, \& Steer, 1988) has many conceptually somatic items such as "feelings of choking," "sweating," and "difficulty breathing" that might be symptoms of anxiety, sleep apnea, or their combination. Because the BAI does not account for the etiology of symptoms, this overlap may result in scores that overestimate the severity of anxiety symptoms. Other authors have also posited that certain somatic items of the BAI may highlight symptoms of medical illness rather than anxiety (Wetherell \& Gatz, 2005). This overlap in symptomatology necessitates psychometric evaluation of the BAI to determine the validity and reliability of the BAI in patients presenting to a sleep disorder center.

The BAI was originally developed with adult psychiatric patients; therefore, the appropriateness of its use in other populations must be tested (Beck et al., 1988). When assessing reliability and validity of a measure, it is also important to examine the latent factor structure of the instrument. In studies that conducted factor analyses on the BAI, the factor structure has varied, ranging from two to six factors, typically consisting of one cognitive factor and one to five somatic factors (e.g., Hewitt \& Norton, 1993; Osman, Barrios, Aukes, Osman, \& Markway, 1993; Wetherell \& Arean, 1997). Because the factor structure of the BAI within a SDB sample has not been previously evaluated and because factor analytic studies vary greatly in their obtained factor solutions, exploratory factor analysis within this sample is warranted.

Furthermore, individual differences can impact scores on the BAI as well as presentation of SDB. For example, obesity is common in patients with SDB, and body mass may partially explain the relation between poor emotional functioning and SDB (Bardwell, Barry, Ancoli-Israel, \& Dimsdale, 1999). Ethnic minorities are at higher risk for obesity and related medical correlates such as apnea (Kumanyika, 2002). Some studies have found younger age to be 
related to higher scores on the BAI (Gillis, Haaga, \& Ford, 1995; Owens, Hadjistavropoulos, \& Asmundson, 2000). There are also gender differences in the presentation and prevalence rates of SDB, with diagnosis being less prevalent in women and women being more likely to present with comorbid depression, insomnia, or hypothyroidism (Hahn \& Staats, 2004; Shepertycky, Banno, \& Kryger, 2005). Hewitt and Norton (1993) examined psychometric properties of the BAI separately for men and women with psychiatric diagnoses and concluded that the factor structure of the BAI (2-factor solution) did not differ by gender but how they responded to items did, with women having higher scores.

The purpose of this study was threefold. First, we aimed to retrospectively collect and examine data on the BAI in an ethnically diverse sample of patients seeking treatment for SDB. In doing so, we planned to document the psychometric properties of the BAI within this sample to promote accurate clinical assessment of anxiety symptomatology in individuals with SDB. We specifically aimed to explore the relation of demographic variables to BAI total scores, including obesity as measured by body mass index (BMI), age, ethnicity, and gender. Second, we aimed to document the psychometric properties of the BAI within a sample with SDB and to do so within gender groups if differences exist. Reliability, validity, and the underlying factor structure as revealed by exploratory factor analysis were examined. Third, we assessed the relation of the BAI scores with apnea severity indexes and other objective polysomnography (PSG) indexes. This is a preliminary and exploratory study to help raise questions about the complexities of measuring anxiety severity in SDB and not to test the usefulness of the BAI as a diagnostic screener.

\section{METHOD}

\section{Participants}

Data were collected from archival data compiled at the Sleep Disorders Center at Methodist Central Hospital in Memphis, TN. We reviewed charts of 303 consecutively presenting cases at the center. These cases included only individuals who received overnight diagnostic PSG. The final sample consisted of 160 men and 143 women aged 19 to 80 years $(M=47.8, S D=13.0)$. The sample was ethnically diverse, with 184 Caucasians, 113 African Americans, 5 Asians, and 1 "other" ethnicity.

At the Methodist Sleep Disorder Center, patients are typically given a preliminary diagnosis of sleep apnea after being evaluated by a sleep specialist for symptoms of snoring, excessive daytime sleepiness, and so forth. They are then referred for PSG for verifying or disproving the preliminary diagnosis. Diagnoses were assigned via the International Classification of Sleep Disorders: 
Diagnostic and Coding Manual (American Sleep Disorders Association, 2005). Excessive daytime sleepiness and other comorbidities (e.g., heart disease or hypertension) are used to determine appropriate treatment pathways. Only 11 of our 303 participants were not diagnosed with sleep apnea after PSG. These 11 participants received the following diagnoses post-PSG: chronic obstructive pulmonary disease $(n=1)$, upper airway resistance syndrome $(n=1)$, narcolepsy $(n=3)$, periodic limb movement disorder (PLMD; $n=2)$, and impaired daytime functioning $(n=4)$. On the BAI, mean scores of these patients did not differ significantly from those diagnosed with sleep apnea. Of the remaining 292 with confirmed sleep apnea, 21 met diagnostic criteria for an additional diagnosis of either upper airway resistance syndrome, PLMD, or narcolepsy post-PSG.

\section{Procedure}

All new patients presenting to the Sleep Disorder Center complete a set of brief psychological inventories, including the BAI and the Beck Depression Inventory-Second Edition (BDI-II; Beck, Steer, \& Brown, 1996), prior to their overnight sleep study. Specific data retrieved included BDI-II total scores, BAI total scores, BAI item scores, age, gender, height, weight, and diagnosis of sleep disorder. Midway through the study we began collecting BDI-II item scores, and these were collected for 115 participants. Also, several variables from PSG were examined to determine severity of SDB. PSG scoring criteria at the Sleep Disorder Center are based on published practice parameters (Kushida et al., 2005) with apnea defined as a respiratory pause that lasts for more than $10 \mathrm{sec}$ and hypopnea defined as a $30 \%$ decrease in amplitude of airflow with either an arousal of $3 \mathrm{sec}$ or more or an oxygen desaturation $>4 \%$. Collected variables included the following: (a) apnea-hypopnea index (the number of apnea and hypopnea episodes per hour sleep), (b) oxygen desaturation nadir, (c) apnea arousal index (the number of arousals per hour caused by apneic events), (d) myoclonic arousal index, and (e) sleep efficiency (total time asleep divided by total time in bed).

\section{Measures}

BAI. The BAI was developed for assessing the severity of anxiety symptoms without confounding anxiety with depression (Beck et al., 1988). It is a selfreport measure with 21 items that are rated on a 4-point scale for the past week, including today. This scale ranges from 0 (not at all) to 3 (severely, I could barely stand it). The total score of all 21 items (range 0-63) provides an estimate of the severity of anxiety symptoms (Beck \& Steer, 1993). Beck et al. (1988) found the BAI to have reasonable convergent and discriminate 
validity, acceptable 1 -week test-retest reliability $(r[81]=.75)$, and high internal consistency $(\alpha=.92)$. The manual includes normative data for individuals with a variety of anxiety disorders (Beck \& Steer, 1993). Osman et al. (1993) also provided means and standard deviations for a nonclinical sample of adults ( $N=225$; women, $n=159$; men, $n=66$ ) from a midwestern community. Finally, the BAI manual considers total scores of 0 through 7 minimal anxiety, 8 through 15 mild anxiety, 16 through 25 moderate anxiety, and 26 through 63 severe anxiety (Beck \& Steer, 1993).

BDI-II. The BDI-II is a 21-item, self-report questionnaire with good reliability and validity designed to measure the severity of depression (Beck et al., 1996). Each item is rated on a 4-point scale ranging from 0 to 3. It has good internal consistency with a coefficient alpha of .92 for psychiatric patients and .93 for college students. The total score of all 21 items (range 0-63) provides an estimate of the severity of depression symptoms. The BDI-II manual considers total scores of 0 through 13 minimal depression, 14 through 19 mild depression, 20 through 28 moderate depression, and 29 through 63 severe depression.

Sleep disorder clinic questionnaire. Participants completed a self-report questionnaire that provided a variety of demographic, medical, and mental health information, including height and weight. Obesity was measured by BMI, which was calculated by dividing weight $(\mathrm{kg})$ by height $\left(\mathrm{m}^{2}\right)$. A BMI greater than 30 is considered obese (Department of Health and Human Services, 2007). Our sample was predominately obese, with a mean BMI of $36.5(S D=9.5)$. This questionnaire also asked participants to rate how frequently they experienced anxiety on the following scale: (a) rarely or never, (b) sometimes, or (c) often. They were also asked to rate the severity of this anxiety on the following scale: (a) mild, (b) moderate, or (c) severe.

\section{Data Analytic Plan}

Our first study aim, to explore the relation of demographic variables to BAI total scores, was examined by estimating Pearson correlations to determine if age or BMI were related to BAI total scores. This was followed by a $2 \times 2$ analysis of covariance (ANCOVA) examining the relation of gender and ethnicity to BAI scores while modeling BMI as the covariate, given the higher risk for obesity in ethnic minority groups. Only African American and Caucasian ethnicities were examined because only 6 people reported other ethnicities, thus limiting meaningful analysis. Discriminant function analyses were used to explore gender differences in item responses on the BAI.

To reach our second study aim, reliability of the BAI was assessed with Cronbach's alpha and item - total score correlations. Support for discriminant 
and convergent validity was explored by conducting Pearson's correlations to examine the relations between BAI scores and other self-report anxiety measures and the BDI-II. Principal axis factoring followed by varimax rotation was used to enter the 21 items from the BAI and the 21 items from the BDI-II into a combined factor analysis. This analytic approach has been previously applied to provide additional support for discriminant validity of the BAI, the idea being that BAI items will load separately from BDI-II items (Beck et al., 1988; Hewitt \& Norton, 1993; Wetherell \& Arean, 1997). Finally, principal axis factor analysis with planned subsequent varimax factor rotation was used to determine the underlying factor structure of the BAI in this sleep-disordered population. If women and men were found to differ on BAI total scores, we planned to examine psychometric properties of the BAI separately by gender.

Our final study aim, to explore the impact of objective PSG variables (e.g., measures of obstructive sleep apnea severity) on BAI scores, was assessed via multiple regression, with BAI total scores entered as the dependent variable. Relations to total score and any existing factor scores were examined separately.

\section{RESULTS}

\section{Study Aim 1: Relation of Demographic Variables to BAI Total Scores}

Age was not correlated with BAI total scores; however, as expected, BMI weakly but positively related to BAI total scores $(r=.19, p<.01)$. Results of a $2 \times 2$ ANCOVA revealed no effect of ethnicity after modeling BMI as a covariate; therefore, all subsequent analyses are based on data pooled over ethnic groups. However, a significant main effect emerged for gender, with women demonstrating significantly higher BAI scores than men, $F(1,301)=$ $24.14, p<.01$. Discriminant analysis revealed that women responded to BAI items differently than men and scored significantly higher than men on all 21 items (Wilk's $\Lambda=0.80, p<.01$ ). Approximately $68 \%$ of the sample was correctly classified by gender based on BAI scores (76.9\% of men; $58.0 \%$ of women). Table 1 presents BAI and BDI-II mean scores and standard deviations for the entire sample and for men and women. Table 2 presents the distribution of scores for women, men, and the total sample according to the classification guidelines provided by Beck and Steer (1993). The mean total scores from psychiatric samples derived from the BAI manual (Beck \& Steer, 1993) and from a community sample of adult volunteers (Osman et al., 1993) are also listed in Table 2 for comparison. 
TABLE 1

Means and Standard Deviations for BAI and BDI-II Total Scores and BAI Scores From Previous Studies

\begin{tabular}{|c|c|c|c|c|c|c|c|c|c|c|c|c|}
\hline \multirow[b]{2}{*}{ Sample } & \multicolumn{3}{|c|}{$B A I$} & \multicolumn{3}{|c|}{$B D I-I I$} & \multicolumn{3}{|c|}{$\begin{array}{l}\text { Osman et al. } \\
(1993)-B A I\end{array}$} & \multicolumn{3}{|c|}{$\begin{array}{c}\text { Beck and Steer } \\
(1993)-B A I\end{array}$} \\
\hline & $n$ & $M$ & $S D$ & $n$ & $M$ & $S D$ & $n$ & $M$ & $S D$ & $n$ & $M$ & $S D$ \\
\hline Total & 303 & 10.45 & 10.03 & 295 & 12.61 & 10.52 & 225 & 11.54 & 10.26 & $160^{\mathrm{b}}$ & 22.35 & 12.36 \\
\hline Men & 160 & 7.40 & 7.94 & 157 & 10.03 & 9.13 & 66 & 8.83 & 6.92 & $157^{\mathrm{a}}$ & 20.53 & 11.86 \\
\hline Women & 143 & 13.87 & 11.01 & 138 & 15.54 & 11.23 & 159 & 12.66 & 11.20 & $236^{a}$ & 24.14 & 13.56 \\
\hline
\end{tabular}

Note. $\quad \mathrm{BAI}=$ Beck Anxiety Inventory; BDI-II = Beck Depression Inventory-Second Edition.

${ }^{\text {a }}$ The combined mean score for women and men in this sample $(n=393)$ was not provided in the BAI manual.

${ }^{\mathrm{b}}$ The total sample score is for an independent clinical sample of men and women presented in the BAI manual $(n=160)$.

TABLE 2

Distribution of BAI Scores by Severity Classification

\begin{tabular}{lccccc}
\hline & Minimal & Mild & Moderate & Severe & \\
Variable & $0-7$ & $8-15$ & $16-25$ & $26-63$ & $n$ \\
\hline Women & $54(37.8 \%)$ & $37(27.2 \%)$ & $29(18.9 \%)$ & $23(16.1 \%)$ & $143(100 \%)$ \\
Men & $103(64.4 \%)$ & $35(21.9 \%)$ & $17(10.6 \%)$ & $5(3.1 \%)$ & $160(100 \%)$ \\
Total sample & $155(51.8 \%)$ & $74(24.8 \%)$ & $47(14.5 \%)$ & $28(8.9 \%)$ & $303(100 \%)$ \\
\hline
\end{tabular}

Note. $\mathrm{BAI}=$ Beck Anxiety Inventory.

\section{Study Aim 2: Psychometric Properties of the BAI}

Internal consistency. Table 3 presents means, standard deviations, and item - total score correlations for the BAI by gender. The BAI had high internal consistency for the total sample $(\alpha=.92)$, for men $(\alpha=.91)$, and for women $(\alpha=.92)$. In men, item scores ranged from a mean of 0.09 (terrified) to 0.67 (unable to relax; indigestion), whereas the total BAI mean score was 7.40 $(S D=7.94)$. Item - total score correlations ranged from 0.28 (feelings of choking) to 0.66 (fear of losing control). In our sample of women, item scores ranged from a mean of 0.27 (terrified) to 1.05 (unable to relax; feeling hot), whereas the total BAI mean score was $13.87(S D=11.01)$. Item - total score correlations ranged from 0.38 (indigestion) to 0.71 (nervous).

Support for convergent and discriminant validity. Discriminant validity was partially tested by correlating BAI total scores with the BDI-II total scores. The BDI-II was significantly related to the BAI $(r=.62, p<.01)$. The self-report questions from the sleep disorder center regarding frequency and 
TABLE 3

Means, Standard Deviations, and Corrected Item-Total Correlations for BAI Items by Gender

\begin{tabular}{llllll}
\hline & \multicolumn{2}{c}{ Men } & & \multicolumn{2}{c}{ Women } \\
\cline { 2 - 3 } \cline { 5 - 6 } BAI Item & $M(S D)$ & $r$ & & $M(S D)$ & $r$ \\
\hline 1. Numbness or tingling & $0.56(.73)$ & .41 & $0.86(.95)$ & .43 \\
2. Feeling hot & $0.53(.83)$ & .43 & $1.05(.94)$ & .45 \\
3. Wobbliness in legs & $0.37(.70)$ & .47 & $0.69(.92)$ & .53 \\
4. Unable to relax & $0.67(.81)$ & .56 & & $1.05(.97)$ & .56 \\
5. Fear of the worst happening & $0.41(.78)$ & .64 & & $0.69(.96)$ & .67 \\
6. Dizzy or lightheaded & $0.42(.72)$ & .59 & & $0.85(.91)$ & .63 \\
7. Heart pounding or racing & $0.32(.60)$ & .57 & & $0.83(.93)$ & .53 \\
8. Unsteady & $0.26(.54)$ & .63 & & $0.70(.89)$ & .62 \\
9. Terrified & $0.09(.42)$ & .55 & & $0.27(.67)$ & .64 \\
10. Nervous & $0.48(.74)$ & .61 & & $0.89(.90)$ & .71 \\
11. Feelings of choking & $0.25(.58)$ & .28 & & $0.50(.79)$ & .51 \\
12. Hands trembling & $0.27(.62)$ & .56 & & $0.49(.79)$ & .55 \\
13. Shaky & $0.23(.55)$ & .63 & & $0.51(.82)$ & .65 \\
14. Fear of losing control & $0.17(.49)$ & .66 & & $0.32(.73)$ & .56 \\
15. Difficulty breathing & $0.57(.79)$ & .55 & & $0.95(.92)$ & .62 \\
16. Fear of dying & $0.20(.61)$ & .45 & & $0.45(.78)$ & .60 \\
17. Scared & $0.21(.53)$ & .60 & & $0.43(.75)$ & .68 \\
18. Indigestion or discomfort in abdomen & $0.67(.82)$ & .40 & $0.96(.96)$ & .38 \\
19. Faint & $0.10(.39)$ & .55 & $0.29(.70)$ & .52 \\
20. Face flushed & $0.17(.46)$ & .52 & $0.43(.70)$ & .54 \\
21. Sweating (not due to heat) & $0.46(.79)$ & .56 & $0.67(.91)$ & .52 \\
\hline
\end{tabular}

Note. $\quad \mathrm{BAI}=$ Beck Anxiety Inventory; men, $n=160$; women, $n=143$.

severity of anxiety significantly correlated with the BAI (frequency, $r=.39, p<$ .01 ; severity, $r=.49, p<.01)$ in partial support of convergent validity. It is interesting to note that the self-report questions also significantly correlated with the BDI-II (frequency, $r=.49, p<.01$; severity, $r=.52, p<.01$ ). However, results of a combined factor analysis to aid in assessing discriminant validity revealed a two-factor solution (parallel analysis), with one factor consisting solely of BDI-II items and the other solely of BAI items. Item 16 (changes in sleeping pattern) on the BDI-II loaded on both factors. The correlation between factors was $r=.66$.

Exploratory factor analyses. To examine the underlying factor structure of the BAI, we used principal axis factoring with varimax rotation for the total sample and separately for men and women. Parallel analysis (Horn, 1965; Zwick \& Velicer, 1986) was used to determine the number of factors to retain; however, interpretability of factors, parsimony, cross-loadings, and strength of factor loadings were also considered. Parallel analysis included 21 items with 
TABLE 4

Factor Loadings for BAI Items for the Total Sample and by Gender

\begin{tabular}{|c|c|c|c|c|c|c|}
\hline \multirow[b]{2}{*}{ BAI Item } & \multicolumn{2}{|c|}{ Total Sample } & \multicolumn{2}{|c|}{ Men } & \multicolumn{2}{|c|}{ Women } \\
\hline & Loading & $h^{2}$ & Loading & $h^{2}$ & Loading & $h^{2}$ \\
\hline 1. Numbness or tingling & 0.55 & 0.30 & 0.56 & 0.32 & 0.53 & 0.28 \\
\hline 2. Feeling hot & 0.57 & 0.33 & 0.58 & 0.33 & 0.52 & 0.27 \\
\hline 3. Wobbliness in legs & 0.65 & 0.42 & 0.63 & 0.40 & 0.63 & 0.40 \\
\hline 4. Unable to relax & 0.68 & 0.46 & 0.68 & 0.46 & 0.65 & 0.42 \\
\hline 5. Fear of worst happening & 0.80 & 0.64 & 0.79 & 0.63 & 0.80 & 0.64 \\
\hline 6. Dizzy or lightheaded & 0.76 & 0.58 & 0.71 & 0.51 & 0.75 & 0.56 \\
\hline 7. Heart pounding or racing & 0.72 & 0.51 & 0.73 & 0.53 & 0.65 & 0.43 \\
\hline 8. Unsteady & 0.80 & 0.63 & 0.81 & 0.65 & 0.74 & 0.55 \\
\hline 9. Terrified & 0.88 & 0.78 & 0.88 & 0.77 & 0.87 & 0.75 \\
\hline 10. Nervous & 0.79 & 0.63 & 0.71 & 0.50 & 0.82 & 0.66 \\
\hline 11. Feelings of choking & 0.58 & 0.33 & 0.40 & 0.16 & 0.63 & 0.40 \\
\hline 12. Hands trembling & 0.72 & 0.52 & 0.71 & 0.50 & 0.69 & 0.48 \\
\hline 13. Shaky & 0.81 & 0.65 & 0.78 & 0.61 & 0.79 & 0.62 \\
\hline 14. Fear of losing control & 0.79 & 0.63 & 0.85 & 0.72 & 0.75 & 0.57 \\
\hline 15. Difficulty breathing & 0.71 & 0.51 & 0.64 & 0.41 & 0.72 & 0.52 \\
\hline 16. Fear of dying & 0.76 & 0.57 & 0.67 & 0.45 & 0.77 & 0.59 \\
\hline 17. Scared & 0.83 & 0.69 & 0.81 & 0.66 & 0.83 & 0.69 \\
\hline 18. Indigestion or discomfort in abdomen & 0.51 & 0.26 & 0.56 & 0.32 & 0.47 & 0.22 \\
\hline 19. Faint & 0.76 & 0.57 & 0.76 & 0.57 & 0.75 & 0.56 \\
\hline 20. Face flushed & 0.72 & 0.52 & 0.71 & 0.51 & 0.68 & 0.47 \\
\hline 21. Sweating & 0.65 & 0.42 & 0.69 & 0.47 & 0.63 & 0.40 \\
\hline Eigenvalue (\% variance explained) & $\begin{array}{c}10.97 \\
(100 \%)\end{array}$ & - & $\begin{array}{c}10.47 \\
(100 \%)\end{array}$ & - & $\begin{array}{c}10.49 \\
(100 \%)\end{array}$ & - \\
\hline
\end{tabular}

Note. $\quad \mathrm{BAI}=$ Beck Anxiety Inventory $\mathrm{h}^{2}=$ communalities.

1,000 iterations. Results suggested a one-factor solution for the total sample and for men and women separately. The one-factor solution produced by parallel analysis is very similar to that of the actual eigenvalues, thus strongly supporting this solution. Independent solutions for men and women were nearly identical to that of the total sample. Table 4 gives the factor loadings for the total sample and for men and women. Again, results were similar across gender and strongly support a one-factor solution including support from parallel analyses, eigenvalue plots, and high internal consistency.

\section{Study Aim 3: Relation of BAI Scores to Apnea Severity Indexes}

The following independent measures of apnea severity were entered into regression analyses for the total sample and separately for men and women: apneahypopnea index, oxygen desaturation nadir, and apnea arousal index. None of 
these severity indexes were significantly related to BAI total scores for men, $F(159)=0.52, p=.67$; women, $F(142)=1.17, p=.32$; or the combined sample, $F(302)=0.92, p=.43$. Other sleep indexes, including sleep efficiency and myoclonic arousal index, were analyzed separately and were also unrelated to BAI total scores. Because factor analyses resulted in single-factor solutions, regressions by factor scores were not completed.

\section{DISCUSSION}

The primary goal of this study was to document psychometric properties of the BAI with a sample from a sleep disorder clinic given concerns about overlapping symptomatology between anxiety and sleep apnea. Overall, psychometric properties of the BAI were acceptable within our SDB population. This supports the use of the BAI as a gauge of anxiety severity in individuals with SDB wherein its usefulness was previously unknown. These results are clinically meaningful in that such assessment may facilitate and inform evaluation and treatment of anxiety within this high-risk population. Specifically, the BAI demonstrated good reliability and high internal consistency. The examination of convergent validity was limited by absence of data from other validated anxiety measures, although BAI scores were significantly related to items on our simple clinic questionnaire examining frequency and severity of anxiety. Despite the high correlation between the BAI and BDI-II, combined factor analysis revealed two distinct factors, suggesting that this correlation is a function of the relation between the two constructs (anxiety and depression) versus poor discriminant validity of the two measures. Some authors have suggested that the BDI and BAI measure two distinct but related factors within the higher order dimension of negative affectivity (Enns, Cox, Parker, \& Guertin, 1998). Within this conceptualization, it is not surprising that the BAI and BDI-II were highly correlated, and yet items proved to load on two distinct factors. Furthermore, factor analyses revealed a simple one-factor solution as opposed to a more complex structure; there was no evidence to support a distinct set of items that appeared to be more related to apnea symptomatology than anxiety. The properties for the total sample also held true for the separate gender groups.

With respect to individual differences, only gender was related to BAI scores after controlling for BMI. Women scored significantly higher than men, as seen in clinical and nonclinical samples from previous studies (Beck \& Steer, 1993; Osman et al., 1993; Osman, Kopper, Barrios, Osman, \& Wade, 1997). BAI scores were examined separately for men and women, and our conclusions correspond with those of Hewitt and Norton (1993). Specifically, the factor structure of the BAI does not seem to differ between men and women, but the response pattern 
to individual items does, with women having higher scores on each item and more variability in scores than men. In fact, discriminant analyses revealed that the way in which women and men respond to individual items differs; therefore, classification into gender groups based on responses was accurate in most cases. Although the prevalence of anxiety diagnosis is known to be higher among women, this observed difference raises questions as to how men and women may experience symptoms of anxiety differently, specifically among individuals with SDB.

Means in our sample were lower than those of psychiatric samples, as expected, yet similar to those of a community sample (Beck \& Steer, 1993; Osman et al., 1993). Almost one quarter of our sample had BAI scores at the moderate or severe level. We would expect that a percentage of these individuals would not meet diagnostic criteria for an anxiety disorder; therefore, our results do not appear to be greatly discrepant from the prevalence rates of anxiety disorders previously reported within individuals with SDB (16.7\%; Sharafkhaneh et al., 2005). Our results did not support a strong relationship between severity of anxiety symptoms and severity of SDB, as BAI scores and PSG apnea indexes were not related. Although external validity is a strength for this study in that data were gathered from a large diverse sample of patients with SDB, several limitations prohibit us from drawing conclusions about the relation of SDB and anxiety. We were only able to present our sample means in relation to those of previously published studies because we could not collect data from a healthy control group. Also, data were collected retrospectively and not all participants received a diagnostic interview to rule-in or rule-out anxiety disorders. Therefore, we did not aim to test the positive and negative predictive ability of the BAI, thus, the usefulness of the BAI as an anxiety disorder screener. We were also unable to present the prevalence of anxiety disorders within our sample. Our hope is that future research will be able to address these issues and continue to elucidate the relation of these two sets of symptoms.

Understanding the relation of anxiety to SDB is possible only to the extent that reliable and valid assessment tools are developed and sound methodology is used. Accurate clinical assessment of anxiety severity might be facilitated by empirical comparisons between existing anxiety measurement tools within SDB samples. For example, McQuaid, Stein, McCahill, Laffaye, and Ramel (2000) reported that the BAI was a relatively poor screening measure for anxiety disorders in a primary care population; however, a modified version of the BAI has been developed specifically for use with primary care populations (BAI-PC) and is reported to have good sensitivity and specificity for identifying panic disorder and generalized anxiety disorder in primary care settings (Beck, Steer, Ball, Ciervo, \& Kabat, 1997). This brief instrument eliminated the somatic and performance items from the original form. The State-Trait Anxiety Inventory (STAI) is another well-established inventory used as an outcome measure in 
a study examining the impact of continuous positive airway pressure (CPAP) treatment on anxiety and depression in individuals with SDB (Sanchez et Al., 2001; Spielberger, 1983). It does not appear that the psychometric properties of either tool have been evaluated in SDB to date; therefore, their merit in comparison to the BAI is unknown. The utility of the BAI, BAI-PC, and STAI as diagnostic screeners in SDB has not been investigated either, and these gaps in the literature may guide future research.

\section{REFERENCES}

American Sleep Disorders Association, Diagnostic Classification Steering Committee. (2005). ICSD International Classification of Sleep Disorders: Diagnostic and coding manual. Westchester, IL: American Academy of Sleep Medicine.

Andrews, J. G., \& Oei, T. P. S. (2004). The roles of depression and anxiety in the understanding and treatment of obstructive sleep apnea syndrome. Clinical Psychology Review, 24, 1031-1049.

Bardwell,W. A., Barry, C. C., Ancoli-Israel, S., \& Dimsdale, J. E. (1999). Psychological correlates of sleep apnea. Journal of Psychosomatic Research, 47, 583-596.

Beck, A. T., Epstein, N., Brown, G., \& Steer, R. A. (1988). An inventory for measuring clinical anxiety: Psychometric properties. Journal of Consulting and Clinical Psychology, 56, 893-897.

Beck, A. T., \& Steer, R. A. (1993). Manual for the Beck Anxiety Inventory. San Antonio, TX: Psychological Corporation.

Beck, A. T., Steer, R. A., Ball, R., Ciervo, C. A., \& Kabat, M. (1997). Use of the Beck Anxiety and Depression Inventories for primary care with medical outpatients. Assessment, 4, 211-219.

Beck, A. T., Steer, R. A., \& Brown, G. K. (1996). Manual for the Beck Depression Inventory (2nd ed.). San Antonio, TX: Psychological Corporation.

Borak, J., Cieslicki, J. K., Koziej, M., Matuszewski, A., \& Zielinski, J. (1996). Effects of CPAP treatment on psychological status in patients with severe obstructive sleep apnoea. Journal of Sleep Research, 5, 123-127.

Department of Health and Human Services, Centers for Disease Control and Prevention. (2007). BMI-Body mass index, 2007. Retrieved February 1, 2007, from http://www.cdc.gov

Enns, M. W., Cox, B. J., Parker, J. D. A., \& Guertin, J. E. (1998). Confirmatory factor analysis of the Beck Anxiety and Depression Inventories in patients with major depression. Journal of Affective Disorders, 47, 195-200.

Gillis, M. M., Haaga, D. A., \& Ford, G. T. (1995). Normative values for the Beck Anxiety Inventory, Fear Questionnaire, Penn State Worry Questionnaire, and Social Phobia and Anxiety Inventory. Psychological Assessment, 7, 450-455.

Hahn, P. Y., \& Staats, B. A. (2004). Differences between men and women in sleep disordered breathing. Journal of Men's Health \& Gender, 1, 312-318.

Haynes, P. L. (2005). The role of behavioral sleep medicine in the assessment and treatment of sleep disordered breathing. Clinical Psychology Review, 25, 673-705.

Hewitt, P. L., \& Norton, G. R. (1993). The Beck Anxiety Inventory: A psychometric analysis. Psychological Assessment, 5, 408-412.

Horn, J. L. (1965). A rationale and test for the number of factors in factor analysis. Psychometrika, 30, 179-185.

Kumanyika, S. K. (2002). Obesity treatment in minorities. In T. A. Wadden \& A. J. Stunkard (Eds.), Handbook of obesity treatment (pp. 416-446). New York: Guilford. 
Kushida, C. A., Littner, M. R., Morgenthaler, T., Alessi, C. A., Bailey, D., Coleman, J., et al. (2005). Practice parameters for the indications for polysomnography and related procedures: An update for 2005. Sleep, 28, 499-519.

McQuaid, J. R., Stein, M. B., McCahill, M., Laffaye, C., \& Ramel, W. (2000). Use of brief psychiatric screening measures in a primary care sample. Depression Anxiety, 12, 21-29.

Osman, A., Barrios, F. X., Aukes, D., Osman, J. R., \& Markway, K. (1993). The Beck Anxiety Inventory: Psychometric properties in a community population. Journal of Psychopathology and Behavioral Assessment, 15, 287-297.

Osman, A., Kopper, B. A., Barrios, F. X., Osman, J. R., \& Wade, T. (1997). The Beck Anxiety Inventory: Reexamination of factor structure and psychometric properties. Journal of Clinical Psychology, 53, 7-14.

Owens, K. M. B., Hadjistavropoulos, T., \& Asmundson, G. J. G. (2000). Addressing the need for appropriate norms when measuring anxiety in seniors. Aging \& Mental Health, 4, 309-314.

Sanchez, A. I., Buela-Casal, G., Bermudez, M. P., \& Casas-Maldonado, F. (2001). The effects of continuous positive air pressure treatment on anxiety and depression levels in apnea patients. Psychiatry and Clinical Neurosciences, 55, 641-646.

Sharafkhaneh, A., Giray, N., Richardson, P., Young, T., \& Hirshkowitz, M. (2005). Association of psychiatric disorders and sleep apnea in a large cohort. Sleep, 28, 1405-1411.

Shepertycky, M. R., Banno, K., \& Kryger, M. H. (2005). Differences between men and women in the clinical presentation of patients diagnosed with obstructive sleep apnea syndrome. Sleep, 28 , 309-314.

Spielberger, C. D. (1983). Manual for the State-Trait Anxiety Inventory (STAI). Palo Alto, CA: Consulting Psychologists Press.

Wetherell, J. L., \& Arean, P. A. (1997). Psychometric evaluation of the Beck Anxiety Inventory with older medical patients. Psychological Assessment, 9, 136-144.

Wetherell, J. L., \& Gatz, M. (2005). The Beck Anxiety Inventory in older adults with generalized anxiety disorder. Journal of Psychopathology and Behavioral Assessment, 27, 17-24.

Zwick, W. R., \& Velicer, W. F. (1986). Comparison of five rules for determining the number of components to retain. Psychological Bulletin, 99, 432-442. 